The Astrophysical Journal, 656:242-247, 2007 February 10

(C) 2007. The American Astronomical Society. All rights reserved. Printed in U.S.A.

\title{
A GLIMPSE OF THE SOUTHERN JELLYFISH NEBULA AND ITS MASSIVE YSO
}

\author{
E. P. Mercer, ${ }^{1}$ D. P. Clemens, ${ }^{1}$ J. M. Rathborne, ${ }^{1}$ M. R. Meade, ${ }^{2}$ B. L. Babler, ${ }^{2}$ R. Indebetouw, ${ }^{3}$ \\ B. A. Whitney, ${ }^{4}$ C. Watson, ${ }^{5}$ M. G. Wolfire, ${ }^{6}$ M. J. Wolff, ${ }^{4}$ T. M. Bania, ${ }^{1}$ R. A. Benjamin, ${ }^{7}$ \\ M. Cohen, ${ }^{8}$ J. M. Dickey, ${ }^{9}$ J. M. Jackson ${ }^{1}$ H. A. Kobulnicky, ${ }^{10}$ J. S. Mathis, ${ }^{2}$ \\ S. R. Stolovy, ${ }^{11}$ B. UzPen, ${ }^{10}$ and E. B. Churchwell ${ }^{2}$ \\ Received 2006 July 14; accepted 2006 October 18
}

\begin{abstract}
In Spitzer/IRAC images obtained under the GLIMPSE Legacy Survey, we have identified a unique and provocative nebular object we call the "Southern Jellyfish Nebula." The Southern Jellyfish Nebula is characterized by a fan of narrow tendrils with extreme length-to-width ratios that emanate from the vicinity of a bright infrared point source embedded in a smaller resolved nebula. From CO observations of the Nebula's morphologically associated molecular cloud, we have derived a kinematic distance of $5.7 \pm 0.8 \mathrm{kpc}$ and a cloud mass of $3.2 \pm 0.9 \times 10^{3} M_{\odot}$. The tendril-like ropes of the Nebula have widths of $\sim 0.1 \mathrm{pc}$ and lengths of up to $\sim 2 \mathrm{pc}$. We have integrated the infrared spectral energy distribution (SED) of the point source to establish it as a massive young stellar object (MYSO), most likely forming alone, but possibly masking fainter cluster members. The shape of the SED is consistent with the shape of a late Class 0 SED model. Based on its far-IR luminosity of $3.3 \pm 0.9 \times 10^{4} L_{\odot}$, the Southern Jellyfish's MYSO has a zero-age main sequence (ZAMS) spectral type of B0. Given the curious nature of this nebula, we suspect its peculiar IR-bright structure is directly related to its current state of star formation.
\end{abstract}

Subject headings: Galaxy: general — H II regions — infrared: ISM — infrared: stars — stars: distances — stars: formation

\section{INTRODUCTION}

Molecular cloud complexes often reveal highly filamentary substructures (Simon et al. 2001). New light is being shed on how stars forming within these filamentary clouds may be organized. Recent studies by Gutermuth et al. (2005) and Teixeira et al. (2006) have found examples of embedded clusters whose protostars are distributed in linear patterns. Furthermore, these elongated features were found to be aligned with the gas and dust filaments of their host molecular clouds. Observations of active star forming sites may provide clues to how such ordered systems arise. Since observations at mid-infrared wavelengths best expose embedded active sites of star formation, it is useful to study the structure and morphology of mid-IR bright star-forming nebulae. These nebulae may provide insight into the intermediate phases of star formation that occur after the collapse and fragmentation of molecular clouds but before stars have shed much of their circumstellar material.

The Galactic Legacy Infrared Mid-Plane Survey Extraordinaire (GLIMPSE; Benjamin et al. 2003; Churchwell et al. 2004) project has revealed an assortment of new star formation regions (SFRs) and stellar clusters in the Milky Way. These SFRs exhibit a broad range of physical characteristics and evolutionary states. Examples of these regions include infrared dark clouds thought to contain protoclusters (Rathborne et al. 2006), young embedded

\footnotetext{
1 Institute for Astrophysical Research, Boston University, Boston, MA.

2 Astronomy Department, University of Wisconsin, Madison, WI.

3 Department of Astronomy, University of Virginia, Charlottesville, VA.

4 Space Science Institute, Boulder, CO.

5 Department of Physics, Manchester College, North Manchester, IN.

6 Department of Astronomy, University of Maryland, College Park, MD.

7 Physics Department, University of Wisconsin-Whitewater, Whitewater, WI.

8 Radio Astronomy Laboratory, University of California, Berkeley, CA.

9 Department of Astronomy, University of Minnesota, Minneapolis, MN. WY.

${ }^{0}$ Department of Physics and Astronomy, University of Wyoming, Laramie,

11 Spitzer Science Center, California Institute of Technology, Pasadena, CA.
}

clusters (Mercer et al. 2005), and high-mass star forming "bubbles" (Churchwell et al. 2006). These recently discovered objects in the GLIMPSE survey provide ideal laboratories for studying the morphology and stellar contents of active star forming regions.

In this paper, we present discovery and follow-up observations of one particular star forming region, whose appearance in the mid-IR is rare and provocative. We have identified an emission nebula in the GLIMPSE data that exhibits features not matched by any other object in the $220 \mathrm{deg}^{2}$ survey area. This nebula, which we call the "Southern Jellyfish Nebula," exhibits several long tendrils of mid-IR emission extending from a bright point source and showing extreme length-to-width ratios.

We identified the Southern Jellyfish Nebula in the four mid-IR GLIMPSE wave bands and analyzed its mid-IR structure. We mapped the nebula using spectral line observations of ${ }^{12} \mathrm{CO}$ and ${ }^{13} \mathrm{CO}$. We also obtained archival data at near- and far-IR wavelengths to ascertain the nature of the nebula and its point source. In the following sections, we present this combined multiwavelength view of the nebula, describe its unique structure, and derive several of its physical properties, including distance, mass, and spectral type of its embedded point source.

\section{OBSERVATIONS}

\subsection{Spitzer Discovery Observations}

Spitzer Space Telescope (Werner et al. 2004) observations were obtained by the Infrared Array Camera (IRAC; Fazio et al. 2004) on 2004 February 11 as part of the GLIMPSE survey. IRAC is a four-channel camera operating simultaneously in wave bands centered on $3.6,4.5,5.8$, and $8.0 \mu \mathrm{m}$ with a $5.21^{\prime} \times 5.21^{\prime}$ field of view (FOV). The integration time for each frame was $1.2 \mathrm{~s}$. The images were processed by the Spitzer Science Center, using version S9.5.0 of the IRAC data processing pipeline. Additional processing of the frames was performed by the GLIMPSE team to remove artifacts and mosaic the images using MONTAGE. The final GLIMPSE image mosaics have pixel sizes of $0.6 \times 0.6 \operatorname{arcsec}^{2}$. 


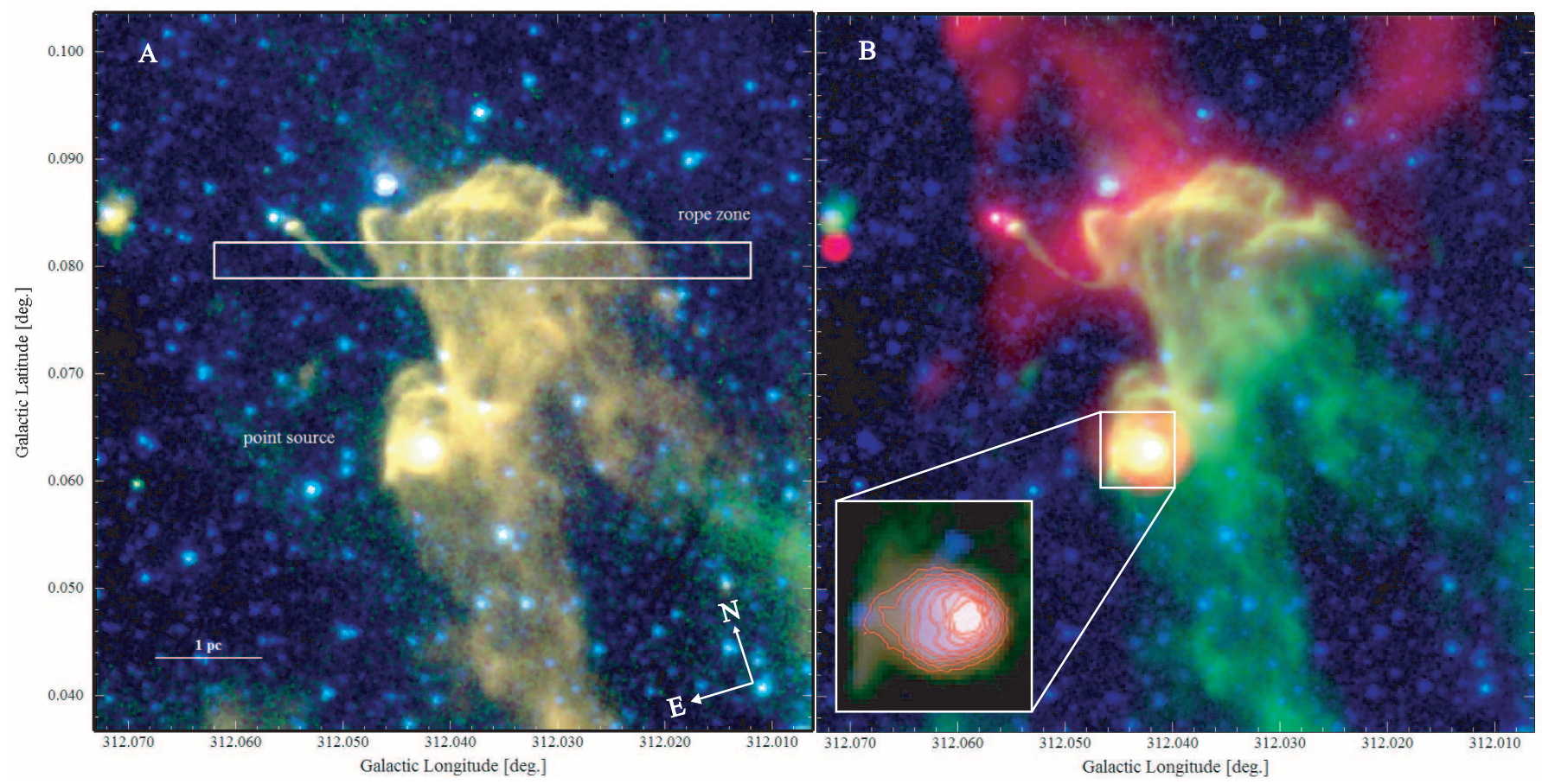

FIG. 1.-(a) Three-color image of the Jellyfish Nebula (white) constructed from IRAC $8.0 \mu \mathrm{m}$ (red), IRAC $5.8 \mu \mathrm{m}$ (green), and IRAC $3.6 \mu \mathrm{m}$ (blue). The rectangle designates the zone over which the variations in fluxes across the ropes were measured for the IRAC and MIPS bands. (b) Three-color image of the Jellyfish Nebula (green) constructed from MIPS $24.0 \mu \mathrm{m}$ (red), IRAC $8.0 \mu \mathrm{m}$ (green), and IRAC $4.5 \mu \mathrm{m}$ (blue). The inset to the lower left shows the bright IR point source embedded in a smaller resolved nebula showing a cavity-like shape.

In all four IRAC images, the Southern Jellyfish Nebula shows a 2 ' fan of mostly parallel "ropes" emanating from the vicinity of a point source, which itself is embedded in a small resolved nebula. Shown in Figure $1 a$ is a three-color image of the Nebula constructed from the IRAC $8.0 \mu \mathrm{m}$ (red), IRAC $5.8 \mu \mathrm{m}$ (green), and IRAC $3.6 \mu \mathrm{m}$ (blue) images. A composite image of the MIPS $24 \mu \mathrm{m}$ (red; observed as part of the MIPSGAL survey by S. Carey et al. 2007, in preparation), IRAC $8.0 \mu \mathrm{m}$ (green), and IRAC $4.5 \mu \mathrm{m}$ (blue) images is shown in Figure $1 b$. The inset image in panel B shows the small resolved nebula, resembling a cavity opening to the left and containing the embedded bright point source. The nebular "ropes" in Figures $1 \mathrm{a}$ and $1 \mathrm{~b}$ are most prominent in the PAH emission-dominated $8.0 \mu \mathrm{m}$ band, but they are seen in all IRAC bands, including in the PAH-free $4.5 \mu \mathrm{m}$ image. In contrast, the MIPS $24 \mu \mathrm{m}$ emission, which traces warm dust, encompasses mainly the northern region of the nebula, as well as the smaller nebula containing the embedded point source to the south.

Profiles of the background-subtracted residual fluxes, drawn from the region highlighted by the white rectangle in Figure $1 a$, are shown in Figure 2. The fluxes were smoothed using a 3 pixel boxcar average. These values for extended apparent emission scale to absolute values when the effective aperture correction factors listed in Reach et al. (2005) are applied. In Figure 2, all four of the IRAC-band profiles clearly show evidence for the ropes, while the MIPS-band profile shows no clear indication of ropes. However, the lower angular resolution of MIPS $\left(6^{\prime \prime}\right)$ would be expected to preclude the detection of the ropes at $24 \mu \mathrm{m}$. The positions of the ropes in the IRAC bands were found by calculating the means of the local maxima in the flux profiles. In total, seven ropes were identified at longitudes: $312.0463 \pm 0.0006$, $312.0446 \pm 0.0003,312.0424 \pm 0.0001,312.0403 \pm 0.0001$, $312.0383 \pm 0.0005,312.0345 \pm 0.0005$, and $312.0327 \pm 0.0003$. The vertical lines in Figure 2 indicate the locations of these ropes, which have approximately uniform spacing of $\sim 7.2^{\prime \prime} \pm 0.5^{\prime \prime}$ (or
$0.20 \pm 0.01 \mathrm{pc}$ at the distance of the Jellyfish, see $\S 3$ ). The mean scatter in the positions of the ropes is $\sim 1.2^{\prime \prime}$, or about 2.5 times the scatter in the spacing of the ropes. The ropes also exhibit a $\sim 40 \%-50 \%$ flux modulation between the rope and interrope positions. The resolved widths of the ropes $\left(\sim 4^{\prime \prime}\right)$ and their long lengths (up to $80^{\prime \prime}$ ) form a $>20: 1$ aspect ratio, which is seen in no other object in the GLIMPSE survey region.

Closer examination of the IRAC profiles reveals two intriguing, wavelength-dependent features of the ropes. The $3.6 \mu \mathrm{m}$ and the PAH-free $4.5 \mu \mathrm{m}$ residual fluxes are shown in Figure 3. These profiles exhibit a similar shape for longitudes lower than

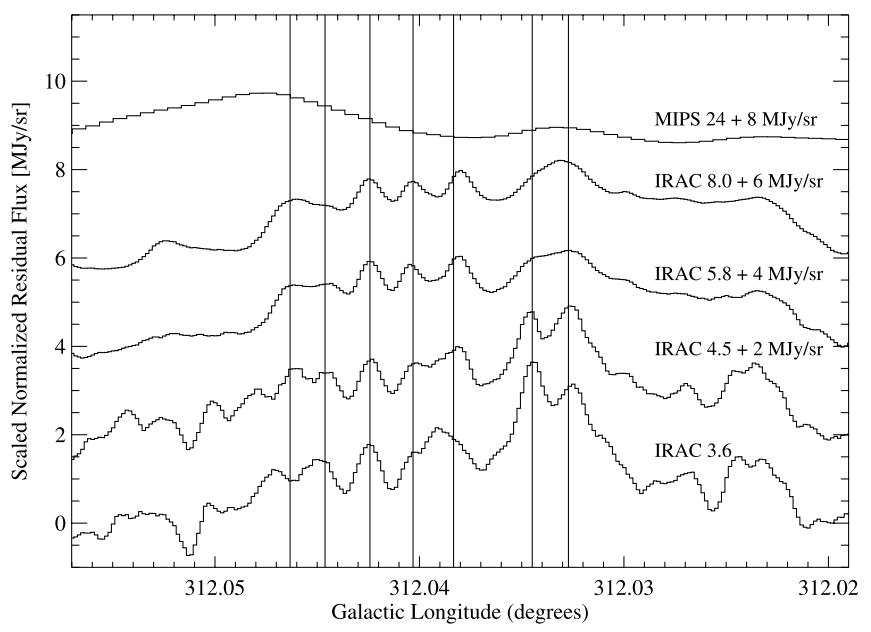

FIG. 2.- Median background-subtracted smoothed flux residuals across the Nebula's ropes, drawn from the rectangular zone in Fig. 1. The 4.5, 5.8, 8.0, and $24 \mu \mathrm{m}$ profiles have been scaled by factors to offset them from the $3.6 \mu \mathrm{m}$ profile. The vertical lines indicate the locations of seven of the ropes, showing a periodiclike space of $\sim 7.2^{\prime \prime} \pm 0.5^{\prime \prime}$ (or $0.20 \pm 0.01 \mathrm{pc}$ ). 


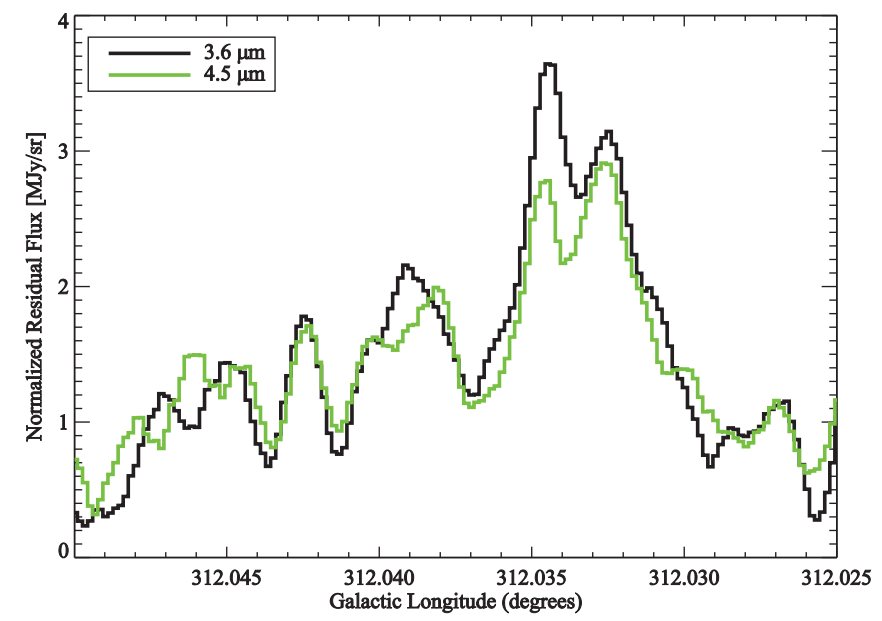

FIG. 3.-Profiles of the 3.6 and $4.5 \mu \mathrm{m}$ median background-subtracted smoothed flux residuals. The $3.6 \mu \mathrm{m}$ profile shows single ropes near 312.039, 312.046 , and 312.048 , where the $4.5 \mu \mathrm{m}$ profile shows two ropes.

312.037. At higher longitudes, deviations arise between the two bands, specifically near $312.039,312.046$, and 312.048 . Toward each of these positions, the $4.5 \mu \mathrm{m}$ profile shows two wellseparated ropes, while the $3.6 \mu \mathrm{m}$ profile shows single ropes located between the two $4.5 \mu \mathrm{m}$ ropes.

A similar comparison between the $4.5 \mu \mathrm{m}$ profile and the PAHdominated 5.8 and $8.0 \mu \mathrm{m}$ profiles indicates further variations of the rope structure with wavelength. In Figure 4, the 5.8 and $8.0 \mu \mathrm{m}$ profiles are highly correlated over the full longitudinal range. These profiles also display similar shapes to that of the $4.5 \mu \mathrm{m}$ profile, but differ markedly near longitudes of 312.034 and 312.045 . Toward these positions, two well-separated ropes are seen at $4.5 \mu \mathrm{m}$, but single broad ropes are seen at 5.8 and $8.0 \mu \mathrm{m}$. All seven of the ropes are cleanly separated in the PAH-free profile. In the three PAH bands, however, some ropes appear to become blended or unresolved. The possible physical or chemical explanations for the observed structural differences in these ropes is unknown.

\subsection{Follow-Up Mopra Millimeter Observations}

New radio spectral line mapping observations were obtained using the Australia Telescope National Facility $22 \mathrm{~m}$ Mopra radio telescope located near Coonabarabran, Australia, on 2005 June $20{ }^{12}$ The $3 \mathrm{~mm}$ SIS mixer receiver was tuned to $110 \mathrm{GHz}$ to observe the ${ }^{13} \mathrm{CO} J=1 \rightarrow 0$ transition and to $115 \mathrm{GHz}$ for the ${ }^{12} \mathrm{CO} J=1 \rightarrow 0$ transition. At these frequencies, the Mopra telescope has a beam size of $33^{\prime \prime}$ and beam efficiencies of $\eta_{110 \mathrm{GHz}}=$ $0.44 \pm 0.03$ and $\eta_{115 \mathrm{GHz}}=0.42 \pm 0.03$ (Ladd et al. 2005). The beam efficiencies were used to convert the antenna temperatures to main beam brightness temperatures. On-the-fly (OTF) mapping was performed to image a $5^{\prime} \times 5^{\prime}$ FOV centered on the Jellyfish. Scans along Galactic longitude were carried out for both of the $\mathrm{CO}$ line transitions. The data cubes were reduced using the AIPS++ LIVEDATA and GRIDZILLA packages as adapted for Mopra data.

We see a potential position error in our data as a $\sim 33^{\prime \prime}$, or 1 beam size offset between the strongest ${ }^{13} \mathrm{CO}$ and ${ }^{12} \mathrm{CO}$ emission features. There are two possibilities for this. First, data collected at Mopra between 2005 May and August 9 using OTF mapping were affected by small (few arcseconds) position time-stamp errors. Second, our ${ }^{12} \mathrm{CO}$ data appear partially contaminated by weak emission in the off positions. Relative off positions, instead of a fixed single

12 The Mopra telescope is part of the Australia Telescope, which is funded by the Commonwealth of Australia for operation as a National Facility managed by CSIRO.

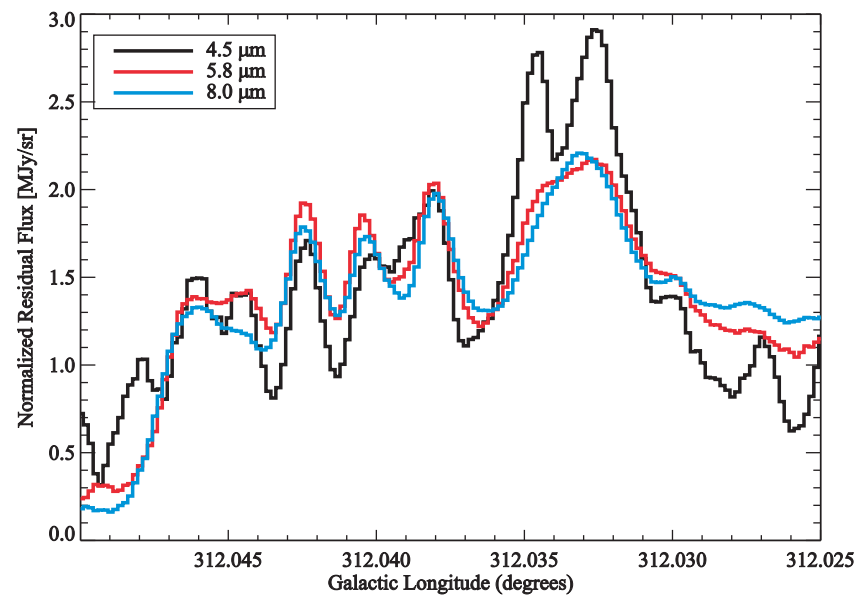

FIG. 4.- Profiles of the 4.5, 5.8, and $8.0 \mu \mathrm{m}$ median background-subtracted smoothed flux residuals. The 5.8 and $8.0 \mu \mathrm{m}$ profiles show a single rope near 312.034 , where the $4.5 \mu \mathrm{m}$ profile shows two ropes. At 5.8 and $8.0 \mu \mathrm{m}$, broad ropes are seen toward 312.045, whereas two ropes are clearly separated at $4.5 \mu \mathrm{m}$.

off, were used during the OTF mapping, which could consequently add apparent structure to the ${ }^{12} \mathrm{CO}$ emission. To resolve the potential time-stamp/position error, the ${ }^{13} \mathrm{CO}$ and ${ }^{12} \mathrm{CO}$ data were individually Gaussian smoothed using a $15^{\prime \prime}$ kernel and their offsets updated by cross-correlating with the IRAC $5.8 \mu \mathrm{m}$ image. This did not affect the generally good morphological matches, but places limits on detailed interpretation at the $30^{\prime \prime}$ level.

The Mopra ${ }^{12} \mathrm{CO}$ and ${ }^{13} \mathrm{CO}$ data cubes were searched in the spectral dimension over the sky field containing the Southern Jellyfish and found to reveal a single spectral feature $\left(V_{\mathrm{LSR}} \sim\right.$ $-60 \mathrm{~km} \mathrm{~s}^{-1}$ ) associated with a compact molecular cloud coincident with the IRAC emission and well detached from any other $\mathrm{CO}$ emission. The data cubes were then integrated over the LSR velocity range from -56.9 to $-63.2 \mathrm{~km} \mathrm{~s}^{-1}$. Figure 5 shows the nebula at $5.8 \mu \mathrm{m}$ with contours of integrated intensity from the

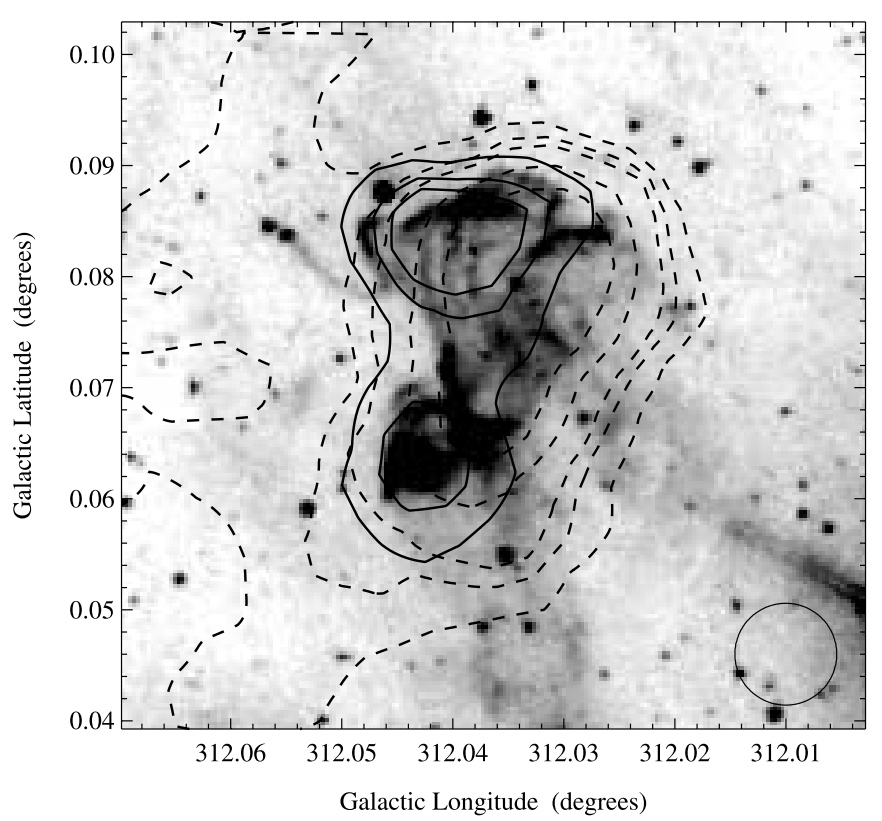

FIG. 5.-IRAC $5.8 \mu \mathrm{m}$ image (inverted gray scale) of the Jellyfish Nebula with ${ }^{13} \mathrm{CO}$ contours (solid) and ${ }^{12} \mathrm{CO}$ contours (dashed) of integrated intensity. The ${ }^{13} \mathrm{CO}$ contours are 5,8 , and $10 \mathrm{~K} \mathrm{~km} \mathrm{~s}^{-1}\left(\sigma_{13 \mathrm{CO}}=0.8 \mathrm{~K} \mathrm{~km} \mathrm{~s}^{-1}\right)$, and the ${ }^{12} \mathrm{CO}$ contours are $5,8,10,15$, and $20 \mathrm{~K} \mathrm{~km} \mathrm{~s}^{-1}\left(\sigma_{12 \mathrm{CO}}=2.7 \mathrm{~K} \mathrm{~km} \mathrm{~s}^{-1}\right)$. The Mopra FWHM beam size is shown at the lower right. The contours were drawn from the Gaussian-smoothed ${ }^{13} \mathrm{CO}$ and ${ }^{12} \mathrm{CO}$ data. See note in text regarding position errors. 
${ }^{12} \mathrm{CO}$ emission (dashed contours) and ${ }^{13} \mathrm{CO}$ emission (solid contours). The $\mathrm{CO}$ appears concentrated into two "cores": one near the northern tendrils, and one associated with the point source in the south.

The ${ }^{12} \mathrm{CO}$ data were tested for evidence of an outflow. First, a composite ${ }^{12} \mathrm{CO}$ spectrum was computed to guide determination of the velocity ranges for potential wing components. The line center velocity of the composite spectrum was determined from a Gaussian fit. The "blue wing" search zone spanned -52.8 to $-55.8 \mathrm{~km} \mathrm{~s}^{-1}$ and the "red wing" from -62.7 to $-65.7 \mathrm{~km} \mathrm{~s}^{-1}$. For all sky positions, the integrated intensity in the red and blue zones were computed. The histograms of the integrated intensities in these zones revealed a narrow blue distribution centered on $-0.2 \pm 0.4 \mathrm{~K} \mathrm{~km} \mathrm{~s}^{-1}$ and a narrow red distribution centered on $0.1 \pm 0.2 \mathrm{~K} \mathrm{~km} \mathrm{~s}^{-1}$. To a $3 \sigma$ upper limit of $\sim 1.4 \mathrm{~K} \mathrm{~km} \mathrm{~s}^{-1}$, these narrow distributions rule out the presence of any strong outflow.

\section{RESULTS}

The ${ }^{12} \mathrm{CO}$ and ${ }^{13} \mathrm{CO}$ line shapes appear very Gaussian and were characterized by fitting single-line Gaussians. From the fits, the ${ }^{13} \mathrm{CO}$ emission tracing the Nebula's host molecular cloud has a peak main beam brightness temperature of $T_{\mathrm{mb}}=15.3 \pm 1.3 \mathrm{~K}$, a mean LSR velocity of $V_{\mathrm{LSR}}=-59.84 \pm 0.10 \mathrm{~km} \mathrm{~s}^{-1}$, and a mean FWHM line width of $\Delta V=3.1 \pm 0.3 \mathrm{~km} \mathrm{~s}^{-1}$. The ${ }^{12} \mathrm{CO}$ peak emission is $T_{\mathrm{mb}}=25.1 \pm 1.7 \mathrm{~K}$, while the mean $V_{\mathrm{LSR}}=$ $-59.20 \pm 0.03 \mathrm{~km} \mathrm{~s}^{-1}$, and mean $\Delta V=2.7 \pm 0.1 \mathrm{~km} \mathrm{~s}^{-1}$. The ${ }^{12} \mathrm{CO}$ and ${ }^{13} \mathrm{CO}$ line center velocities differ by no more than about $20 \%$ of the ${ }^{13} \mathrm{CO}$ line width.

The measured line center velocity of the $\mathrm{CO}$ emission allowed us to compute the kinematic distance to the Jellyfish. This distance was derived by reflection of the northern hemisphere rotation curve of Clemens (1985) with $\left(R_{0}=8.5 \mathrm{kpc}, \Theta_{0}=\right.$ $220 \mathrm{~km} \mathrm{~s}^{-1}$ ). At a longitude of $312^{\circ}$, the $\mathrm{CO}$ velocities for the Southern Jellyfish locate it at the tangent point with a unique kinematic distance of $5.7 \mathrm{kpc}$. Toward this direction, the difference between the velocity of the nebula and the velocity of the tangent point is $\sim 1 \mathrm{~km} \mathrm{~s}^{-1}$. Potential sources of error for the distance estimate include those introduced by the reflection of the northern rotation curve and the error in the velocity of the tangent point, with the latter dominating the uncertainty. Typically, tangent point uncertainties are $10 \%-15 \%$ of the kinematic distance (Kolpak et al. 2003). Based on northern-sky sources with similar reflected longitudes and velocities listed in Table 1 of Kolpak et al. (2003), we assign an error of $15 \%$ or $0.8 \mathrm{kpc}$ to the distance value for the Southern Jellyfish.

The molecular column densities and total mass were derived following the method outlined in Simon et al. (2001), which assumes LTE cloud conditions, optically thick ${ }^{12} \mathrm{CO}$ emission, and optically thin ${ }^{13} \mathrm{CO}$. The ${ }^{13} \mathrm{CO}$ and molecular hydrogen column densities were calculated to be $2.3 \pm 0.2 \times 10^{16} \mathrm{~cm}^{-2}$ and $1.3 \pm 0.1 \times$ $10^{22} \mathrm{~cm}^{-2}$, respectively. At a distance of $5.7 \mathrm{kpc}$, the molecular cloud has dimensions $3.4 \times 5.3 \mathrm{pc}^{2}$. Together, the column density and cloud size yield a cloud mass for the Southern Jellyfish of $3.2 \pm 0.9 \times 10^{3} M_{\odot}$, which has been corrected for the abundances of helium and heavier elements.

The point source at the bottom of the Southern Jellyfish has been detected in previous surveys. It was detected at 12, 25, 60, and $100 \mu \mathrm{m}$ by the Infrared Astronomical Satellite (IRAS), ${ }^{13}$ and is listed as the IRAS point source 14050-6111. The source

\footnotetext{
13 This publication makes use of the NASA/IPAC Infrared Science Archive, which is operated by the Jet Propulsion Laboratory, California Institute of Technology, under contract with NASA.
}

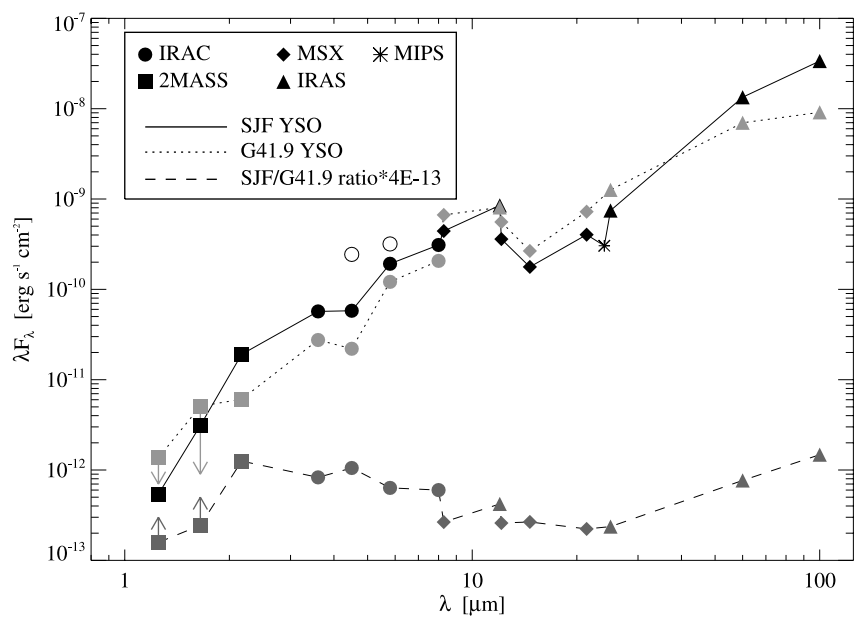

FIG. 6.-SED comparison of the Southern Jellyfish YSO (solid black line connecting black symbols) and the massive YSO found toward G41.9 (Mercer et al. 2004). IRAC and MIPS fluxes were obtained by aperture photometry. IRAC 4.5 and $5.8 \mu \mathrm{m}$ fluxes listed in the GLIMPSE point source archive are indicated by open circles. The dashed curve is the ratio of the Southern Jellyfish YSO SED divided by the G41.9 MYSO SED (offset from the other curves for clarity). Upper and lower limits are indicated by arrows for the 2MASS $J$ - and $H$-band fluxes in the G41.9 SED and ratio curve.

was also detected by the Midcourse Space Experiment (MSX) at $8.38,12.13,14.65$, and $21.30 \mu \mathrm{m},{ }^{14}$ and in the near-IR $J, H$, and $K_{s}$ bands by the Two Micron All Sky Survey (2MASS). ${ }^{15}$ More recently, the source was detected at $24 \mu \mathrm{m}$ in the MIPSGAL survey data.

We developed the point source spectral energy distribution (SED) shown in Figure 6 by combining the 2MASS, GLIMPSE/ IRAC, $M S X$, MIPS, and IRAS data for the point source. The four IRAC fluxes and the MIPS $24 \mu \mathrm{m}$ flux were extracted by performing aperture photometry. For each band, a one-dimensional Gaussian was fit to each flux profile through the point source. Based on the fit, the aperture radius was defined as the number of pixels out to $3 \sigma$ away from the mean. For the 3.6, 4.5, 5.8, and $8.0 \mu \mathrm{m} \mathrm{IRAC}$ bands, these radii were $5,4,7$, and 8 pixels (or $\left.3.0^{\prime \prime}, 2.4^{\prime \prime}, 4.2^{\prime \prime}, 4.8^{\prime \prime}\right)$, respectively. A radius of 4 pixels or $9.6^{\prime \prime}$ was used for the $24 \mu \mathrm{m}$ MIPS image. The inner and outer radii for the sky annuli were set to the $3 \sigma$ star radius and 2 times this value, respectively, in order to capture the surrounding nebular plus background emission and remove it from the point source flux to the full extent possible. Our new aperture photometry yielded an IRAC $8 \mu \mathrm{m}$ flux that is $70 \%$ as bright as the MSX $8 \mu \mathrm{m}$ flux for the point source. Similarly, the MIPS $24 \mu \mathrm{m}$ flux is $40 \%$ as bright as the IRAS $25 \mu \mathrm{m}$ flux. Based on these modest flux decrements measured for the higher angular resolution IRAC and MIPS bands, we conclude nebular contamination is only minimally significant for fluxes shortward of $25 \mu \mathrm{m}$.

The IRAC 4.5 and $5.8 \mu \mathrm{m}$ flux values drawn from the GLIMPSE point source archive are also shown in the SED. The 3.6 and $8.0 \mu \mathrm{m}$ data points are not listed in the GLIMPSE point source archive. Most likely, the source appears extended at these wavelengths because of the small surrounding nebula, and therefore, does not meet the point source extraction criteria.

\footnotetext{
${ }^{14}$ This publication makes use of data products from the Midcourse Space Experiment.

${ }^{15}$ This publication makes use of data products from the Two Micron All Sky Survey, which is a joint project of the University of Massachusetts and the Infrared Processing and Analysis Center/California Institute of Technology, funded by NASA and NSF.
} 
In Figure 6, the SED for the Southern Jellyfish point source is compared to the SED for a massive YSO (G41.9) found toward $l=42^{\circ}$ during the in-orbit checkout (IOC) phase of the GLIMPSE survey (Mercer et al. 2004). In both cases, the SED rises from the near-IR through the mid-IR and continues rising to the last IRAS point at $100 \mu \mathrm{m}$. Because the SED of G41.9 was shown to closely resemble the SED of a model late Class 0 YSO (Whitney et al. 2003), we believe the Southern Jellyfish YSO is also at this same evolutionary state.

Figure 6 also shows the ratio of the SED for the Southern Jellyfish YSO divided by the SED of the G41.9 YSO. This ratio shows an apparent excess in the near-IR as well as a cooler far-IR spectrum for the Southern Jellyfish. The cooler far-IR may signal youth, or at least a more optically thick envelope around the protostar. Nebular contamination likely contributes to this cooler excess since the longer wavelength IRAS bands have much lower resolution. The near-IR excess on the other hand may be produced by scattered light, strong line (or PAH feature) emission, or fainter hotter companions.

The total infrared luminosity of the point source was calculated by integrating the SED and using the CO-based kinematic distance. Two methods were employed to compute the total far-IR flux. Following the method of Helou et al. (1988), the far-IR flux integrated from 1 to $1000 \mu \mathrm{m}$ for a thermally dominated source peaking between 50 and $100 \mu \mathrm{m}$ is $\sim 3.2 \times 10^{-11} \mathrm{~W} \mathrm{~m}^{-2}$. Using the method of Emerson (1988), the integrated flux from 7 to $135 \mu \mathrm{m}$ is $\sim 3.3 \times 10^{-11} \mathrm{~W} \mathrm{~m}^{-2}$. Since these two methods result in similar fluxes, we adopt the mean value before computing the luminosity of the Southern Jellyfish point source. At a distance of $5.7 \mathrm{kpc}$, the luminosity of the source, $L=4 \pi d^{2} \mathrm{~F}$, is $3.3 \pm$ $0.9 \times 10^{4} L_{\odot}$, once all values have been converted to the appropriate units. The spectral type for the YSO was estimated using this far-IR luminosity. Using Table 1 of Martins et al. (2005), a main-sequence star of this luminosity would be of type B0. Since a YSO of this same spectral type would evolve onto the main sequence nearly horizontally from the pre-main-sequence track, a pre-main-sequence B0 would exhibit most of its ZAMS luminosity. Given that warmed circumstellar material may contribute to the total luminosity for this MYSO, attributing total system luminosity entirely to the main-sequence stellar luminosity is maybe an overestimate. In the extreme case of an order-of-magnitude overestimate of the point-source luminosity (i.e., $90 \%$ of the luminosity arises from extended warm dust or PAHs), the resulting ZAMS luminosity of $3.3 \times 10^{3} L_{\odot}$ would still predict a young star of spectral type B3.

An analysis of the IRAC images sensitivity was carried out to estimate the flux limits for each band. The sensitivity limit of each IRAC band was estimated as the $5 \sigma$ limiting flux, which was converted from the magnitude detection limit listed in Table 2 of the GLIMPSE Quality Assurance document (Churchwell et al. 2005) using the IRAC zero-point flux (Reach et al. 2005). The IRAC fluxes for Vega (spectral type A0 V; Reach et al. 2005), scaled to the distance of the Southern Jellyfish, lie just above the detection thresholds. At $3.6 \mu \mathrm{m}$, the flux of Vega scaled to $5.7 \mathrm{kpc}$ is $4.3 \times 10^{-13} \mathrm{ergs} \mathrm{s}^{-1} \mathrm{~cm}^{-2}$ and the sensitivity limit is $2.5 \times 10^{-13} \mathrm{ergs} \mathrm{s}^{-1} \mathrm{~cm}^{-2}$. Hence, our observations are sensitive enough to have detected all stars earlier than $\sim \mathrm{A} 0 \mathrm{~V}$.

At $5.7 \mathrm{kpc}$, the nearest two stars to the bright southern point source lie $\sim 0.3 \mathrm{pc}$ away (the faint blue stars in the inset of Fig. 1b). The GLIMPSE point source archive lists the brighter of these two as $4.5 \mathrm{mag}$ fainter than the bright point source at $4.5 \mu \mathrm{m}$. The mass-luminosity relation predicts this to be a star no earlier than a B6 V, if at the distance of the Southern Jellyfish.
The early-type status and associated youth of these neighboring stars would permit them to be members of a currently forming cluster. However, unlike the nearby MYSO, they appear free of any embedding circumstellar material. These two stars could be more distant and intrinsically luminous (e.g., K giants) or foreground and of lower luminosity. Other lower mass companions to this massive YSO may be present, but are either unresolved and masked by the MYSO emission, or have spectral types below our sensitivity thresholds.

\section{DISCUSSION}

The physical natures of the IR-bright ropes and the interrope emission of the Southern Jellyfish are currently unknown. Several scenarios arise that may explain the appearance of the ropes: (1) confinement of matter by magnetic fields, (2) formation via instabilities, (3) illumination of scattering surfaces, (4) excitation of spectral line emission features, or (5) projection effects.

The mid-IR bright ropes could be revealing the true distribution of gas and dusty matter in the nebula, with very little matter located in the interrope regions. Such a matter-traced distribution could favor a magnetic scenario, with strong magnetic fields either confining matter to the ropes or allowing matter to dissipate along the long axes of the ropes. Alternatively, matter-traced ropes could be the result of an instability, whether magnetically influenced or not (e.g., a shear flow instability).

Another possible explanation is that the bright ropes do not faithfully represent the true distribution of nebular matter, but instead highlight the surfaces from which infrared light is scattered or emitted into our line of sight. Under this scenario, the ropes could represent either real light emitting regions, perhaps dominated by spectral line or PAH emission features, or continuum scattering surfaces. Finally, the bright ropes we see could be the projection onto a more uniform screen of a "scene" illuminated by the YSO through some "picket fence" or mask of extinction near the illuminator. Precessing YSOs can carve patterns into their outflow cavities, although the backfill timescale is likely shorter than the precession timescale for a YSO outflow (Quillen et al. 2005). Perhaps light passing through etched cavities will appear on a nearby, mostly uniform screen as striations or ropes.

Distinguishing among these scenarios requires determining the nature of the infrared light coming from the nebular ropes and interrope regions to ascertain whether we are seeing scattered light (and if so, from which source), intrinsic line or spectral feature emission, and whether the nebula is predominantly neutral, molecular, or arising from a photodissociation region. These await future observations.

The mid-IR morphology of the Southern Jellyfish Nebula is intriguing, and while its shape is peculiar, recent studies may indicate that it need not be unique. Observational studies have shown that stars sometimes appear to form in linear or "spoke"like patterns (Lada et al. 2004; Gutermuth et al. 2005; Teixeira et al. 2006). Spitzer observations of the young cluster NGC 2264 reveal several linear configurations of protostars (Teixeira et al. 2006). In a study of three other young clusters, Gutermuth et al. (2005) found that stars still embedded in their parental molecular clouds appear to form along elongated zones that follow the distributions of the molecular gas. The Southern Jellyfish Nebula, with its MYSO seemingly forming alone and the filamentary ropes emanating from its massive protostar, is perhaps a precursor to these examples of linear star forming structures. The evident star formation taking place at the upper left end of the highest longitude rope in Figures $1 a$ and $1 b$ may also relate to these issues. 


\section{SUMMARY}

We have identified a rare and provocative nebula in the GLIMPSE survey data showing several tendril-like ropes with 20:1 length-to-width ratios emanating from the vicinity of a massive young stellar object. The mid-IR bright Southern Jellyfish Nebula is morphologically associated with a molecular cloud. From CO radial velocity measurements, we determined a kinematic distance of $5.7 \pm 0.8 \mathrm{kpc}$ to the nebula and a cloud mass of $3.2 \pm 0.9 \times 10^{3} M_{\odot}$. The bright, late Class 0 protostar seen anchoring the tendrils has a far-IR luminosity of $3.3 \times \pm 10^{4} L_{\odot}$ and ZAMS spectral type of B0.

The nature of the Nebula's ropes remains unknown, but scenarios involving instabilities and magnetic fields are certainly possible. The detection of a single massive young stellar object is also a mystery, as we might have expected to find members of a forming cluster. It seems likely more stars will form, since the host molecular cloud does contain enough material to produce additional stars. With its resemblance to the spokelike configurations of some distributions of embedded protostars, the Southern Jellyfish Nebula is perhaps the first example of a mid-IR bright nebula experiencing linear-type star formation.

We thank the anonymous referee for carefully reading the paper and thoughtful suggestions that improved its quality. Support for this work, part of the Spitzer Space Telescope Legacy Science Program, was provided by NASA through contracts 1225025 (BU), 1224653 (UWI), 1224988 (SSI), 1224681 (UMD), 1256801 (UWI-W), 1242593 (UCB), 1253153 (UMN), and 11253604 (UWY) issued by the Jet Propulsion Laboratory, California Institute of Technology under NASA contract 1407.
Benjamin, R. A., et al. 2003, PASP, 115, 953

Churchwell, E., et al. 2004, ApJS, 154, 322 2005, in GLIMPSE Quality Assurance Document (Madison: Dept.

Astron., Univ. Wisconsin) 2006, ApJ, 649, 759

Clemens, D. P. 1985, ApJ, 295, 422

Emerson, J. P. 1988, in Formation and Evolution of Low-Mass Stars, ed. A. K. Dupree \& M. T. V. T. Lago (NATO ASI Ser. C, 241; Dordrecht: Kluwer), 193 Fazio, G. G., et. al. 2004, ApJS, 154, 10

Gutermuth, R. A., Megeath, S. T., Pipher, J. L., Williams, J. P., Allen, L. E., Myers, P. C., \& Raines, S. N. 2005, ApJ, 632, 397

Helou, G., Khan, I. R., Malek, L., \& Boehmer, L. 1988, ApJS, 68, 151

Kolpak, M. A., Jackson, J. M., Bania, T. M., Clemens, D. P., \& Dickey, J. M. 2003, ApJ, 582, 756

Lada, C. J., Muench, A. A., Lada, E. A., \& Alves, J. F. 2004, AJ, 128, 1254

\section{REFERENCES}

Ladd, N., Purcell, C., Wong, T., \& Robertson, S. 2005, Publ. Astron. Soc. Australia, 22, 62

Martins, F., Schaerer, D., \& Hillier, D. J. 2005, A\&A, 436, 1049

Mercer, E. P., et al. 2004, ApJS, 154, 328 2005, ApJ, 635, 560

Quillen, A. C., Thorndike, S. L., Cunningham, A., Frank, A., Gutermuth, R. A., Blackman, E. G., Pipher, J. L., \& Ridge, N. 2005, ApJ, 632, 941

Rathborne, J. M., Jackson, J. M., \& Simon, R. 2006, ApJ, 641, 389

Reach, W. T., et al. 2005, PASP, 117, 978

Simon, R., Jackson, J. M., Clemens, D. P., Bania, T. M., \& Heyer, M. H. 2001, ApJ, 551, 747

Teixeira, P. S., et al. 2006, ApJ, 636, L45

Werner, M. W., et al. 2004, ApJS, 154, 1

Whitney, B. A., Wood, K., Bjorkman, J. E., \& Cohen, M. 2003, ApJ, 598, 1079 